Le patrimoine archivistique et documentaire équin de la Manche : constitution et reconstitution; construction et valorisation des patrimoines et réciproquement

\title{
Alain Talon
}

\section{CpenEdition}

\section{Journals}

Édition électronique

URL : http://journals.openedition.org/insitu/9642

DOI : $10.4000 /$ insitu.9642

ISSN : 1630-7305

Éditeur

Ministère de la culture

Référence électronique

Alain Talon, « Le patrimoine archivistique et documentaire équin de la Manche : constitution et reconstitution ; construction et valorisation des patrimoines et réciproquement », In Situ [En ligne], 18 | 2012, mis en ligne le 31 juillet 2012, consulté le 20 avril 2019. URL : http:// journals.openedition.org/insitu/9642 ; DOI : 10.4000/insitu.9642

Ce document a été généré automatiquement le 20 avril 2019

\section{(i) $\odot$

In Situ Revues des patrimoines est mis à disposition selon les termes de la licence Creative Commons Attribution - Pas d'Utilisation Commerciale - Pas de Modification 4.0 International. 


\title{
Le patrimoine archivistique et documentaire équin de la Manche : constitution et reconstitution ; construction et valorisation des patrimoines et réciproquement
}

\author{
Alain Talon
}

1 Le 20 novembre 1881, Elisa meurt de vieillesse.

1881, 20 novembre, dimanche,

Ma pauvre vieille et bonne jument Élisa, la mère de Conquérant, la source première et féconde de nos trotteurs de Normandie est morte ce matin. En revenant de la Messe, je l'ai retrouvée étendue raide, près de l'entrée du jardin Gouey qu'elle avait brisée en tombant. Je l'avais mise dans cette petite pièce bien abritée pour qu'elle y put passer commodément l'hiver. Je me suis penché sur ma pauvre jument, une longue larme mouillait encore sa paupière, comme si sa dernière larme avait été pour moi. Involontairement j'ai senti mes yeux se mouiller.

Élisa est morte de vieillesse, presque sans souffrance sans doute, elle ne pouvait avoir une meilleure fin? Je l'ai soignée dans ses derniers ans comme elle l'avait mérité. Je n'ai pas été ingrat envers elle et je l'ai traitée comme une amie. Sa noble carrière est terminée et je ne puis plus rien pour elle que de lui donner un regret.

Elle est enterrée entre les deux noyers du Clos Grenneville ${ }^{1}$. 
Figure 1

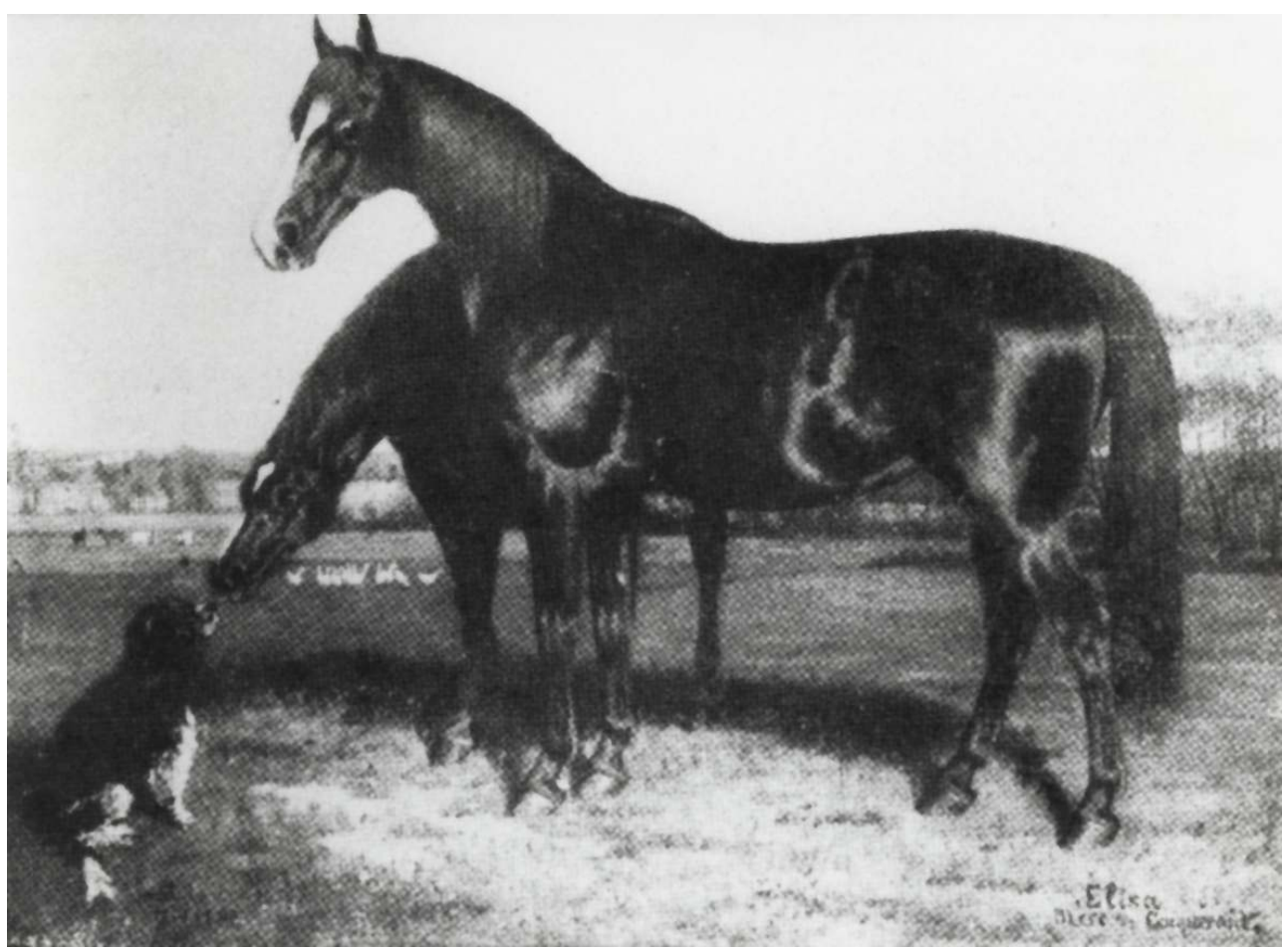

La jument Élisa, mère de Conquérant, peinture de Paul Le More, copie d'une reproduction dans le Trotteur de France, n91, 28 décembre 1989.

Phot. A. Poirier, collection privée. (C) AD Manche/CG50.

2 L'auteur de cette lettre émouvante, l'éleveur Pierre-Étienne Joseph-Lafosse (1828-1897) ${ }^{2}$ (fig. $\left.\mathbf{n}^{\circ} \mathbf{1}\right)$ (fig. $\mathbf{n}^{\circ}$ 2) de Saint-Côme-du-Mont, a connu la gloire d'avoir fait naître Élisa la grande aïeule de la race trotteuse. Il a aussi eu la peine de la voir mourir. Ce témoignage extraordinaire d'un événement triste, illustre la relation de l'homme au cheval et du cheval à l'homme. Et c'est là l'un des enjeux des patrimoines équestres et équins. Au-delà de la connaissance, de l'étude du cheval dans ses composantes si diverses, c'est la connaissance de l'homme, de son activité, de son goût ou de son dégoût pour le cheval, de ses activités pour et autour du cheval qui présente un intérêt majeur. 
Figure 2

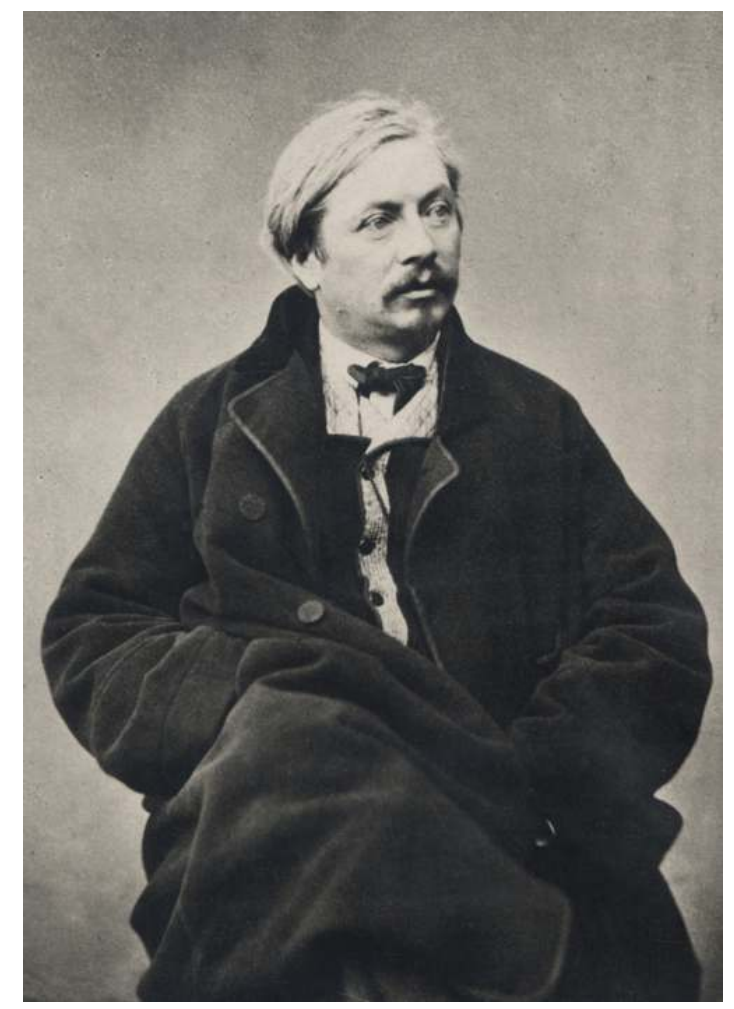

Pierre-Étienne Joseph-Lafosse de Saint-Côme-du-Mont (1828-1897).

Phot. A. Poirier, collection privée. (C) AD Manche/CG50.

Activités économiques, sciences hippiatriques et vétérinaires, productions artistiques, littéraires (on pourrait ajouter cette lettre de Joseph-Laffosse à l'une des nombreuses anthologies du cheval), activités militaires qui renvoient dans un passé relativement récent au rôle, à l'œuvre des Haras nationaux ; activités sportives, là aussi militaires puis civiles, civiles et mixtes ( $80 \%$ des cavaliers sont des cavalières), relation nouvelle de l'homme au cheval qui passe du statut d'animal de rente à celui d'animal de compagnie.

4 Aussi, il n'y a pas un pan de notre société passée où le cheval n'ait joué un rôle, une fonction, de la plus ingrate (la plupart des travaux) à la plus sacralisée (sépulture). De cette omniprésence historique - de laquelle devraient découler de multiples travaux de recherches qui restent à écrire - il suffit de lire le premier tome de "la culture équestre » de Daniel Roche ${ }^{3}$ pour sonder les pans de cette histoire à écrire, cette " histoire réelle " du cheval selon la formule de Jean-Pierre Digard ${ }^{4}$ pour qui «le peu qui a déjà été fait demeure à peu près totalement ignoré du public ». De cette omniprésence dans l'histoire du cheval découlent des patrimoines multiformes, matériels (immobilier, mobilier) et immatériels (savoir, savoir-faire, traditions, usages et règles).

Le département de la Manche est un département très concerné par cette histoire et ces patrimoines, de manière très significative depuis la Révolution. Très concerné car pour des raisons au moins géographiques, ce département est devenu le laboratoire de conception du trotteur; le premier territoire de production du cheval de remonte ${ }^{5}$, du carrossier. Il en résulte depuis la Libération, la création d'une zone de tout premier ordre pour le cheval de sport: pays naisseur et formateur de jeunes chevaux, pays de grands compétiteurs, cavaliers et chevaux et également pour le cheval de courses (trotteurs). 
6 La Manche est par conséquent très intéressée par la connaissance, la conservation du patrimoine équin résultant de ces activités. Ainsi, la constitution, la gestion patrimoniale de l'ensemble de ces matériaux apparaissent en toute logique essentielles pour la valorisation culturelle, économique, la connaissance de ce département.

7 Intéressé, enfin, car ce département est aussi l'un, si ce n'est le premier touché par les destructions, notamment archivistiques lors des combats de la libération de la France. Les archives départementales et ses collections ont ainsi été détruites le 6 juin 1944 (fig. n³). Depuis les années cinquante, une politique archivistique particulière a été conçue afin de pallier ces destructions, afin de «reconstituer» le patrimoine documentaire de ce département.

Figure 3

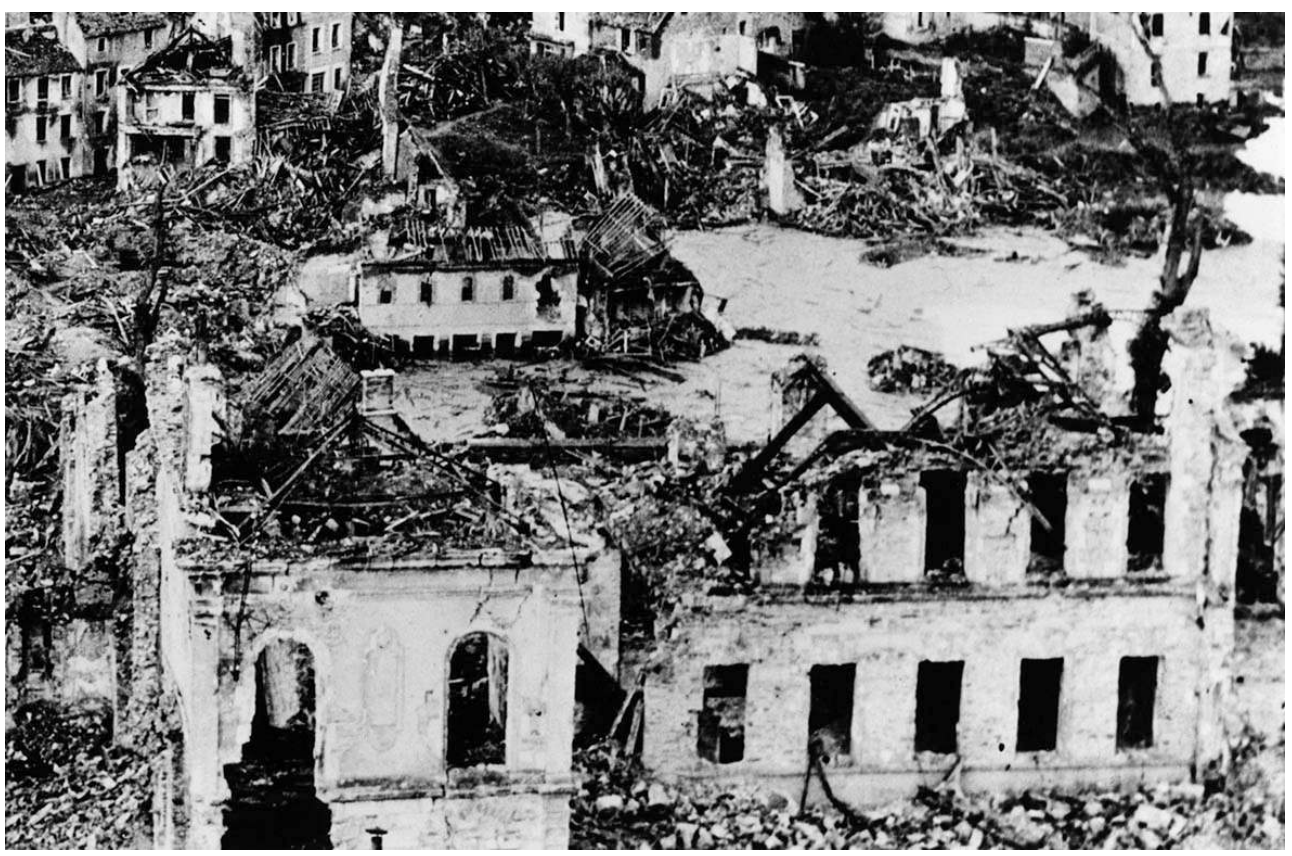

Service des Archives de la Manche détruit le 6 juin 1944.

Phot. A. Poirier, 2 Num 2001/325. ( ) AD Manche/CG50.

8 À ce titre, le patrimoine écrit des haras en constitue l'une des composantes majeures et aussi l'un des instruments de cette politique de reconstitution des collections, avec d'autres types de sources.

\section{Constitution du patrimoine archivistique et documentaire}

\section{Les versements des archives du haras de Saint-Lô ${ }^{6}$}

Dans le cadre des missions de collecte des archives publiques, les archives du haras de Saint-Lô ont fait l'objet de plusieurs versements entre 2003 et 2005 alors que la première visite sur site avait été réalisée en 1995. Faute de place, cette prise en charge n'avait pu se faire plus tôt alors que les archives étaient conservées en grande partie (450 mètres linéaires avant traitement) au-dessus de la forge avec les risques d'un sinistre (fig. $\mathbf{n}^{\circ} \mathbf{4}$ ). 
Figure 4

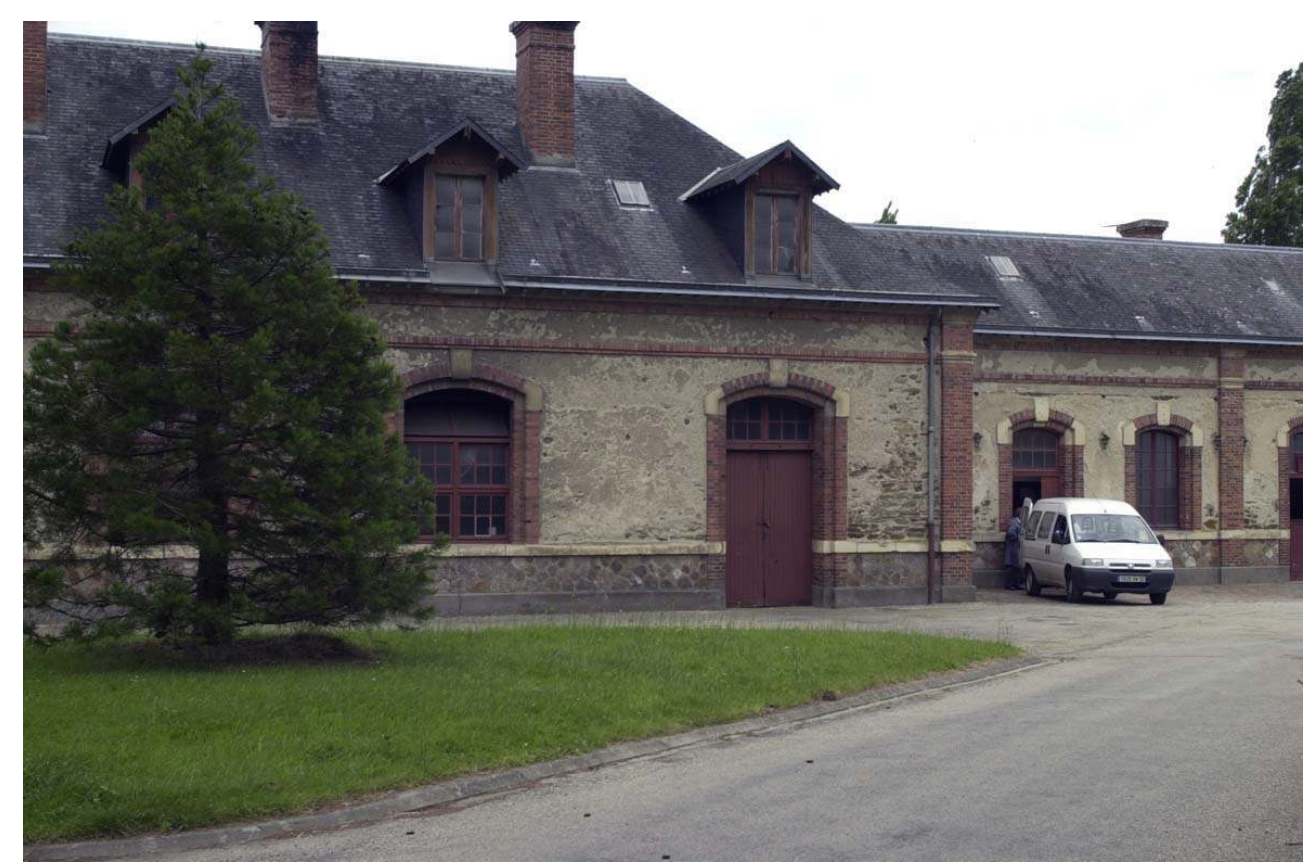

La forge du haras de Saint-Lô, local de conservation des archives modernes, 2003.

Phot. A. Poirier, 3 Num 2003/221. (c) AD Manche/CG50.

10 Cette prise en charge est concomitante à la réforme des Haras nationaux, commencée en 1999. Dans les évolutions de l'institution, le volet patrimonial est officiellement pris en compte, il porte notamment sur les archives. L'instruction de tri élaborée entre 2005 et 2006 et diffusée en janvier 2007 en est pour partie la traduction. Celle-ci dresse un tableau de l'existant avec, comme souvent, des difficultés à mêler les besoins inhérents aux archives définitives, patrimoniales, parfois inconnues de l'administration des Haras, et les documents récents ou contemporains de gestion. Comme pour d'autres administrations prestigieuses où la culture maison est très importante, la conception et surtout la mise en œuvre de cette circulaire en pleine réforme ont pu, localement, être plus ou moins bien perçues.

Le travail de classement du premier versement identifié sous la cote 2 ETP se traduit par la réalisation d'un inventaire de 444 pages « papier » comprenant 1348 articles couvrant la période 1806-2001. Ce fonds représente après reconditionnement près de deux cents mètres linéaires.

En 2005, un versement complémentaire et beaucoup plus modeste de vingt-cinq mètres linéaires est identifié sous la cote 6 ETP. Il comprend 194 articles, pour la période 1922-2001. Il correspond, approximativement à des documents plus récents ou bien à des documents d'archives qui n'avaient pu être appréhendés lors des premières opérations de collecte.

\section{Les enjeux des archives du haras de Saint-Lô}

Pour le cas de Saint-Lô, on retrouve de manière ordinaire la production archivistique d'un dépôt d'étalons dont le ressort géographique englobe tout le département de la Manche et la partie située à l'ouest de l'Orne pour le département du Calvados (fig. $\mathbf{n}^{\circ}$ ). La 
richesse documentaire $\mathrm{du}$ fonds est très importante. Parmi les dossiers ou séries de documents particulièrement intéressants et qui méritent d'être mentionnés, signalons :

14 - La collection des carnets de montes et de saillies qui débute en 1896. Elle est complète depuis 1899 , et rassemble 7500 registres cotés à la pièce par année puis par station de monte (fig. ${ }^{\circ}$ 6). Il s'agit là de l'état civil des chevaux, patrimoine archivistique de premier plan mais aussi patrimoine génétique, pour qui s'intéresse aux documents relatifs aux chevaux, patrimoine économique.

\section{Figure $^{\circ} 5$}

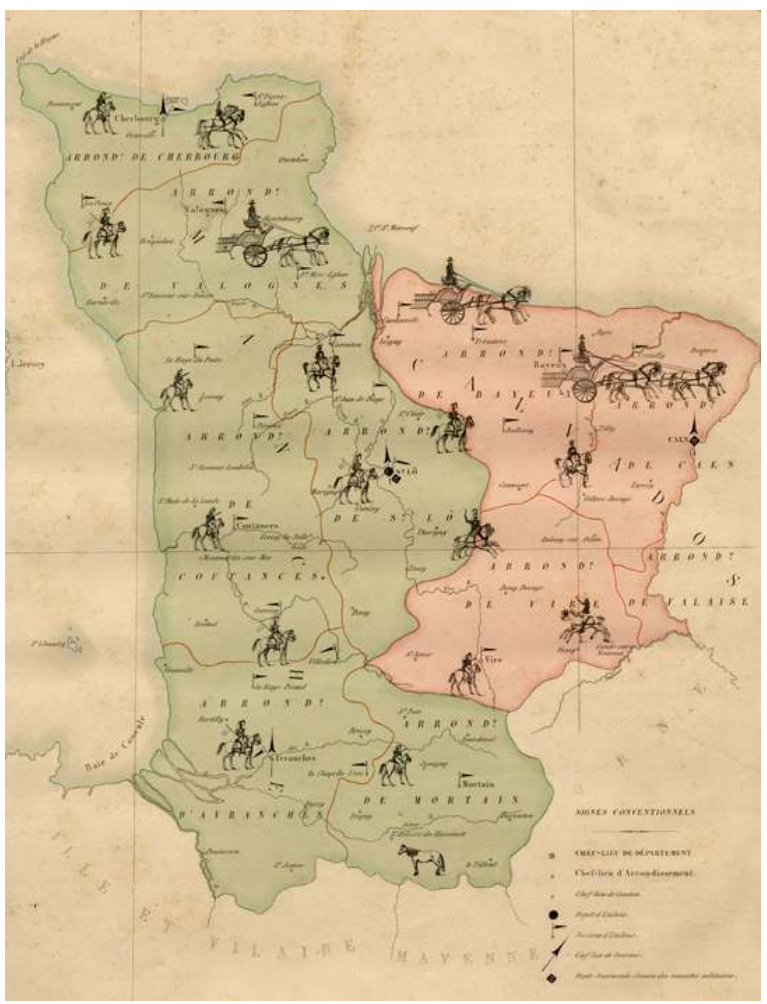

Circonscription du haras de Saint-Lô. GAYOT, Eugène (éd.), LALAISSE, Hippolyte (ill.). Atlas statistique de la production des chevaux en France [...]. Paris : éd. P. Dupont, 1850, AD Manche, BIB RES A 10. 
Figure $^{\circ} 6$

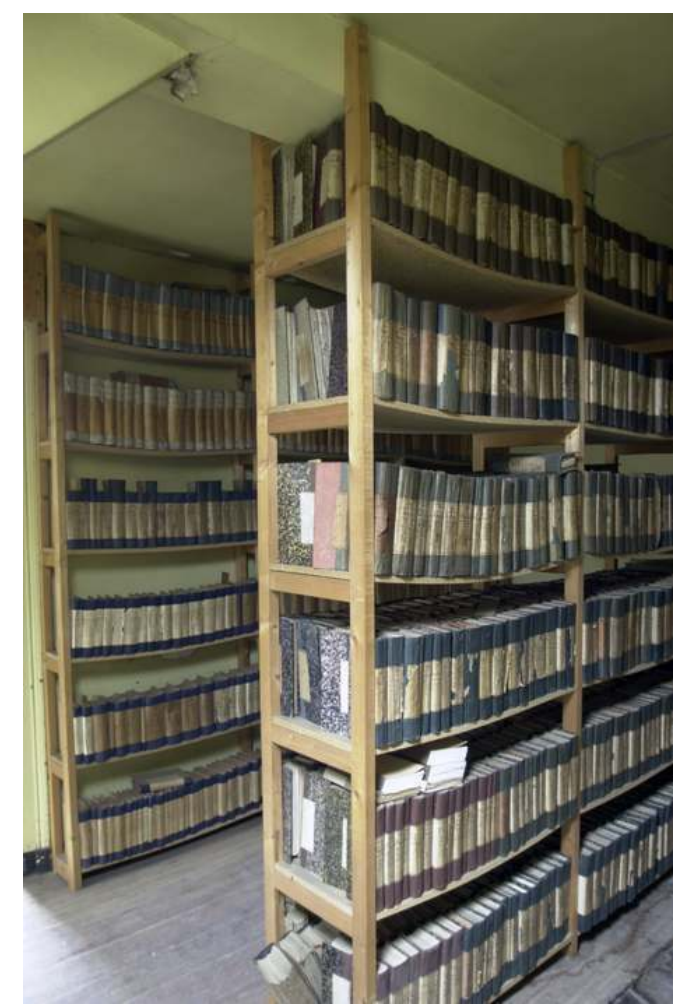

Détail de la collection des 7500 carnets de montes et de saillies conservés au haras de Saint-Lô avant le versement de 2003. Phot. A. Poirier, 3 Num 2003/215. (C) AD Manche/CG50.

15 - La collection des 24 registres d'étalons, ouverte dès la création du Haras en 1806. Elle documente plus de 5500 étalons et permettrait de retracer l'histoire de l'activité du haras, notamment :

- L'évolution de son effectif d'étalons ainsi que sa composition par race ${ }^{7}$ (notamment celle, disparue, du carrossier noir du Cotentin),

- La conception de l'administration des Haras nationaux de l'étalonnage et son pendant du côté des éleveurs, à savoir l'utilisation des chevaux du pays,

- L'évolution du nombre de juments saillies, celle du prix des étalons, du prix des saillies, - L'évolution du réseau géographique des stations de monte ; le rôle et l'influence de chacune d'entre elles dans un département où les pays sont très marqués, tout comme les modèles des chevaux et l'usage qui en découle.

16 - Les rapports des directeurs, à confronter aux rapports des inspections conservés aux Archives nationales sous les cotes $\mathrm{F} / 10$, dupliqués - au titre des microfilms de complément des archives - pour être consultés à Saint-Lô ${ }^{8}$ décrivent l'ensemble des activités du haras.

17 - Les documents à propos des stations de monte qui permettent de documenter un patrimoine immobilier public ou devenu privé, disséminé sur le territoire et dont l'étude reste à mener.

18 - Les dossiers sur les deux guerres mondiales, très importants pour un établissement dont la vocation militaire est primordiale et aussi pour reconstituer un patrimoine par ailleurs détruit. 

archives départementales, les fonds épargnés offrent un intérêt primordial au titre de la politique de reconstitution des collections. Les archives du haras en sont l'un des exemples. En 1944, les bombes ont détruit une grande partie du site du haras ${ }^{9}$, alors que les archives, conservées dans le bâtiment à droite de l'entrée principale, ont été épargnées, à quelques mètres près (fig. $\mathbf{n}^{\circ} 7$ ).

Figure 7

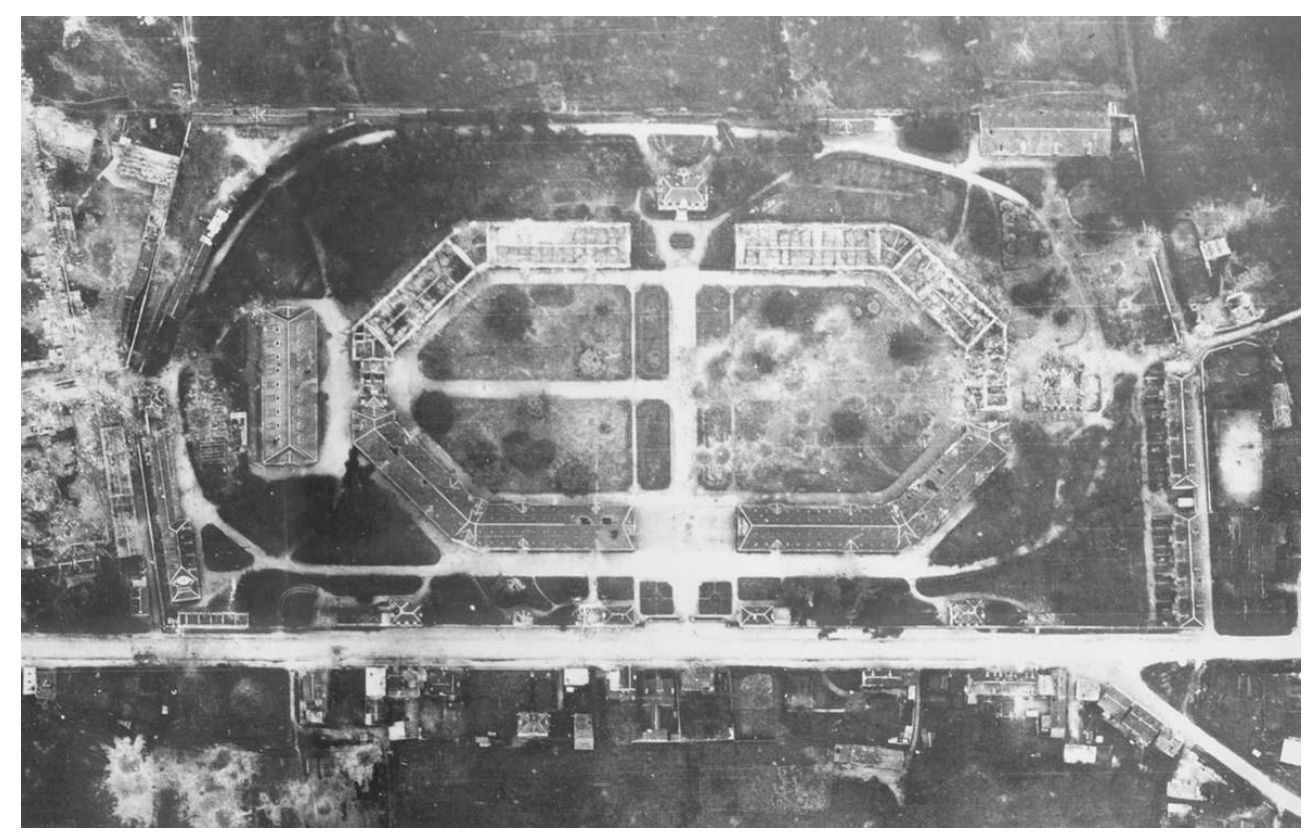

Le Haras de Saint-Lô après les bombardements, c. juillet 1944.

(C) Arch. nat. Américaines.

In Situ, 18 | 2012 


\section{Reconstitution du patrimoine archivistique} certes contribué à beaucoup de pertes, notamment en 1944, mais aussi à des phases de reconstruction et de reprise en main sur le plan administratif par les différents pouvoirs notamment civil et religieux. Ces réorganisations administratives sont prégnantes après 1450, après les guerres de Religion, peut-être après la Révolution (mais c'est plus difficile à vérifier). Sur le plan archivistique, cela se manifeste par une forte densité de fonds d'archives privées et également de chartriers ${ }^{10}$.

\section{La collecte des archives privées}

31 Des années soixante au début des années quatre-vingt-dix, une campagne de collecte de fonds familiaux et privés a délibérément été engagée. Elle a permis le dépôt voire le don ou le prêt pour microfilmage de complément de fonds essentiels pour la reconstitution des collections. Cela se vérifie ainsi ponctuellement pour les archives inhérentes à la gestion des chevaux, conservées parmi les chartriers, les archives familiales. 

historique. Ce sont souvent des fonds d'érudits, tels que :

- le fonds Alfred Butot (145 J 13) qui concerne notamment le cheval, son usage militaire au Moyen Âge, son commerce au XVe et XVI siècle.

- le fonds Paul de Gibon ( $213 \mathrm{~J}$ ) qui intéresse l'élevage du cheval pendant le premier XX siècle, la création de différentes sociétés de courses de la région (dont celle de Jullouville, de Saint-Pair), l'association des propriétaires de chevaux de France et des colonies, le projet d'Union bas-normande et percheronne, 1889-1905.

\section{Figure 8}

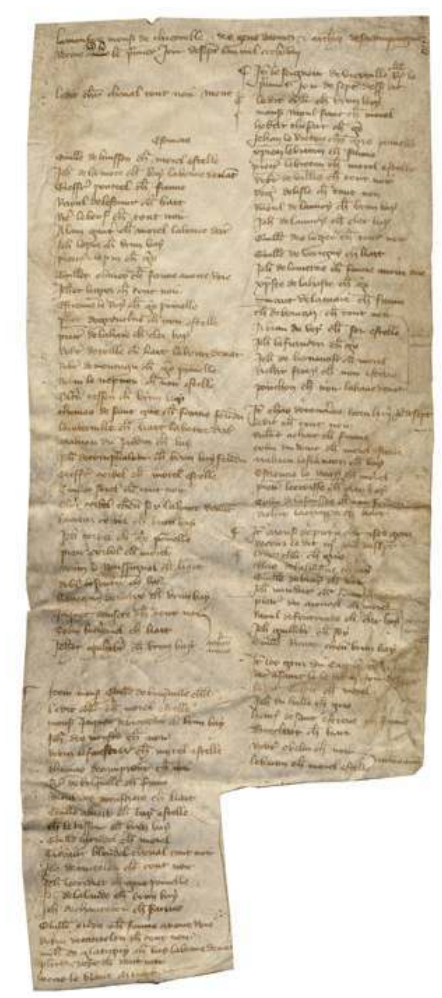

Montre du seigneur de Thieuville, des gens d'armes et archers de sa compagnie de Saint-Lô pour le roi de France, Charles V, 1368. AD Manche, 2 J 1054 [parchemin 42,5 x $18 \mathrm{~cm}$ ].

Un certain nombre de pièces isolées, multiformes et achetées intéressent différents aspects de l'histoire du cheval, par exemple :

- 2 J 1054 : montre des gens d'armes de Saint-Lô, 1368 (fig. nº) : illustration même du document d'archives dont l'intérêt documentaire est très éloigné du contexte originel qui a justifié sa production. Ce document nous fournit des informations précises sur la robe des chevaux. Celle-ci, mentionnée comme moyen de reconnaissance des gens d'armes à solder, nous apporte des éléments quantitatifs et qualitatifs précieux pour l'identification des chevaux utilisés au Moyen-Âge ${ }^{12}$. Jusqu'au XIX ${ }^{e}$ siècle, la couleur de la robe du cheval est un critère d'appréciation, un outil de sélection pour l'élevage et l'amélioration des races. À ce titre, (un beau et) un bon cheval doit être de couleur baie foncée voire noire. 
- $5 \mathrm{~J} 88$ : recueil sur l'art vétérinaire des chevaux : manuscrit avec dessins et planches aquarellées, fin XVIII ${ }^{e}$ siècle. Ce traité signé de «Claude-François Canut dit La Joye, soldat au régiment d'Enghien $»\left(\right.$ fig. $\left.\mathbf{n}^{\circ} \mathbf{9}\right)$ représente une sorte de condensé des connaissances populaires en hippiatrie.

Figure 9

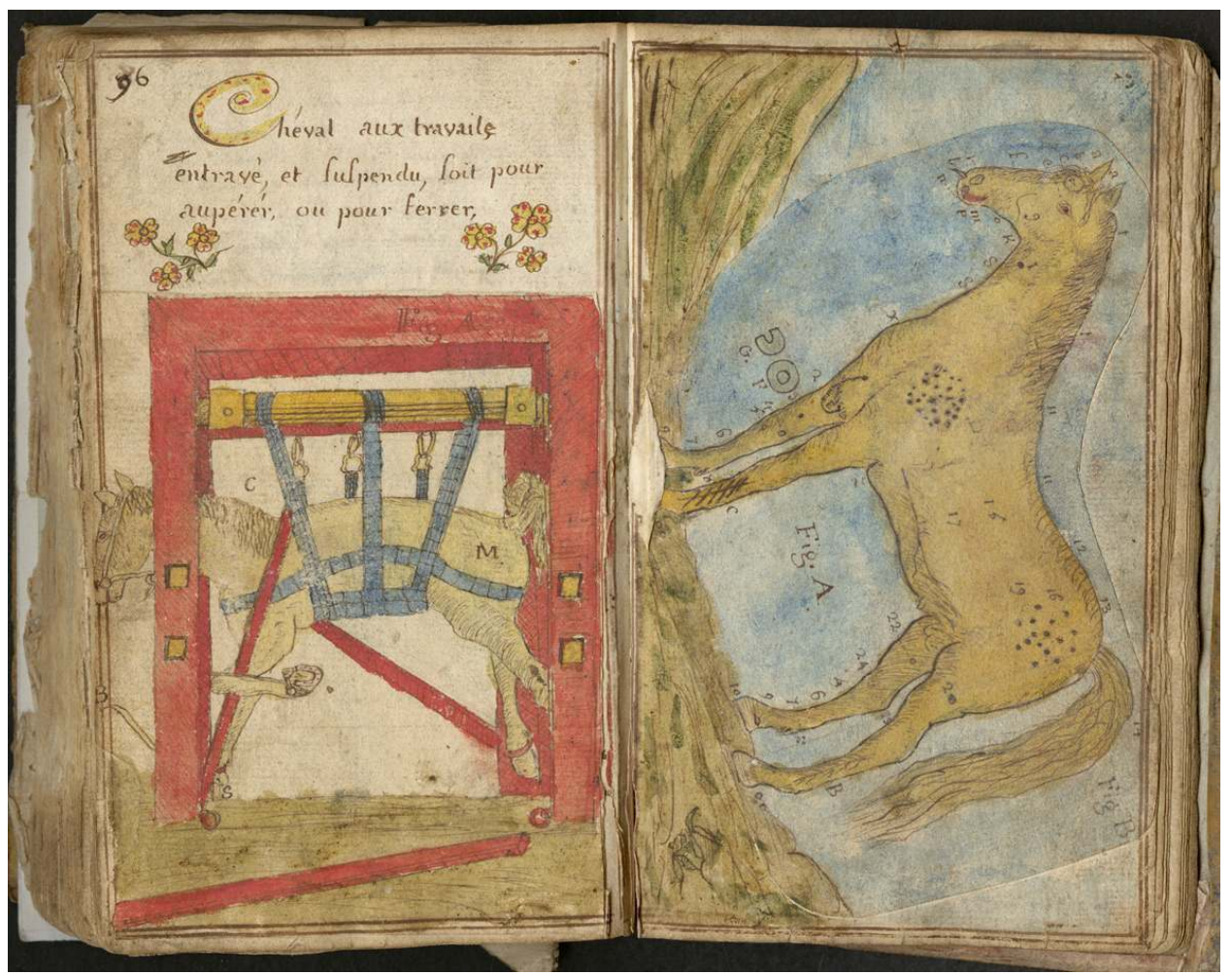

Recueil sur l'art vétérinaire des chevaux : manuscrit avec dessins et planches aquarellés par « ClaudeFrançois Canut dit La Joye, soldat au régiment d'Enghien », fin XVIII siècle. AD Manche, $5 \mathrm{~J} 88$ [papier $17 \times 11 \mathrm{~cm}]$.

Certaines pièces amalgamées à des fonds classés parmi les archives privées fournissent des informations très précieuses sur le cheval. Par exemple, parmi les riches archives de la baronnie de Bricquebec ${ }^{13}$, on trouve un registre de comptabilité de l'abbaye du Vœu de Cherbourg tenu par le prieur Combeaux entre 1759 et 1774 (280 J 250). Ce document couvre l'ensemble des dépenses de l'établissement monastique. On peut ainsi relever les frais relevant de l'utilisation, de l'entretien des chevaux, les modes de transport : location de chevaux de selle, frais de carrosse, parcours, durée et coût des voyages, etc.

Ce dernier exemple illustre le caractère atomisé des sources sur l'histoire du cheval au sein des archives privées. On peut ainsi relever au hasard d'un contentieux dont les pièces sont conservées dans tel chartrier, des informations sur la mise aux herbages des bovins et des équins ${ }^{14}$, leur exploitation, leur commercialisation.

Les archives publiques offrent bien évidemment des avantages similaires et presque le même éparpillement. Il n'est pas question ici de faire un état des sources publiques sur un tel sujet. Citons simplement le cas des archives judiciaires parfois difficiles à appréhender: elles peuvent également être très riches d'informations sur le mode d'élevage, sur les types de chevaux élevés. Ainsi la collection des procès-verbaux des gardes forestiers de Bricquebec fournit-elle de précieuses informations sur le mode 
d'élevage des chevaux, leurs descriptions, les vols dont ils font l'objet aussi pendant la Révolution au sein d'une forêt confisquée comme bien national ${ }^{15}$. Les constats relevés par les gardes, notamment le mode de pacage des chevaux n'auraient sûrement pas décontenancé le sieur de Gouberville, deux siècles et demi plus tôt, et ses pratiques d'élevage extensif où une grande partie du « haras » est laissée en liberté dans la forêt de Brix $^{16}$.

\section{L'iconographie : photographies et affiches}

La collecte de l'iconographie dont le potentiel documentaire est important procède également de cette politique de reconstitution. Ces documents sont achetés, déposés, donnés, prêtés pour numérisation.

\section{Les photographies}

Il y a une sorte de concomitance entre le développement de la photographie et l'âge d'or de l'usage du cheval, comme animal de transport, de travail, pour les civils, les militaires, les populations rurales et urbaines, pour les travaux agricoles, les travaux liés aux activités du littoral, les activités de commerce. Ainsi la représentation du cheval à travers ces différentes utilisations est omniprésente dans les fonds photographiques conservés et qui couvrent principalement la période entre 1870 et 1960.

Certaines collections concernent plus particulièrement la thématique du cheval :

- la collection de photographies sur les chevaux de courses de trot et de galop

(27 Fi/67 Num), éditées sous forme de cartes. Ce fonds, acheté en mars 2005, comprend 331 pièces de 1875 à 1914.

- le fonds Marguerite Daguenet donné en 2006 et coté en 11 Fi, comprend 748 articles de la fin du XIX et du début du XXe siècle dont une très belle série sur l'ancien dépôt de remonte pour partie sinistré en 1944 et complètement détruit dans le cadre de la Reconstruction de la ville de Saint-Lô.

- d'autres fonds photographiques collectés depuis soixante ans comme les fonds Dufour pour la côte ouest du département (17 Fi), Lefrançois pour le nord ouest (32 Num), Jouenne pour le centre du département ( $31 \mathrm{Fi})$. Ces fonds, antérieurs à la seconde guerre mondiale, documentent la vie en milieu rural, notamment les travaux agricoles pour lesquels le cheval est omniprésent.

- un certain nombre des 60000 cartes postales anciennes dont 42000 sont numérisées et accessibles sur le site internet des archives : http://recherche.archives.manche.fr/

\section{Des affiches, des placards}

41 Une centaine d'articles documente les courses de chevaux depuis le XIX ${ }^{\mathrm{e}}$ siècle (fig. $\mathbf{n}^{\circ} \mathbf{1 0}$ ) 


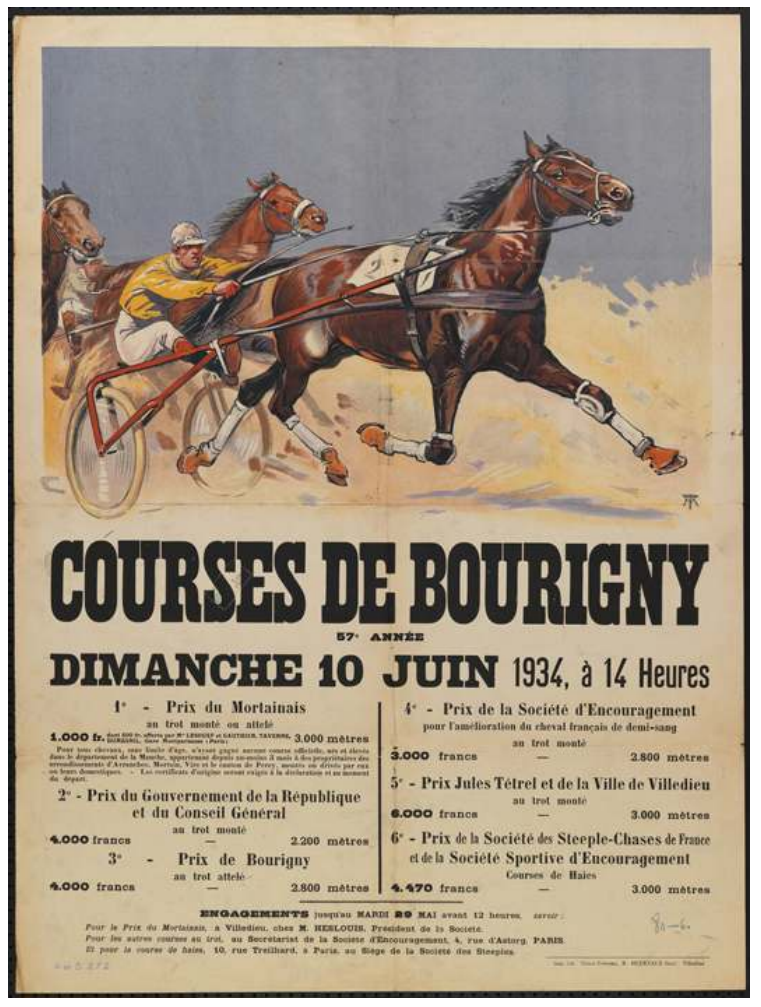

Courses de Bourigny [c. Boisyvon], 12 mai 1935. AD Manche, 200 Fi 1/87 [estampe, (affiche-texte), en couleur].

Par ailleurs, la collection de placards au sein du fonds du haras de Saint-Lô (200 pièces) est très importante pour l'histoire du cheval militaire et également pour les concours d'élevage.

\section{Les imprimés}

43 Les archives de la Manche œuvrent pour la constitution, à des fins patrimoniales, d'une bibliothèque concernant l'histoire et le patrimoine de la collectivité. Les publications antérieures à 1944 contribuent aussi à reconstituer et à enrichir le corpus documentaire sur le sujet. Le fonds (une hippothèque selon le mot de Jérôme Garcin ${ }^{17}$ ) comprend ainsi un certain nombre de monographies traitant: d'hippiatrie, de l'histoire de l'élevage, du cheval de guerre, du cheval de trait, du cheval de trot, du cheval de selle (d'aucuns diront que cela tourne à l'hippomanie, selon la formule de Jean-Louis Gouraud ${ }^{18}$ ). Un effort particulier porte sur l'acquisition des livres anciens autour de ces deux derniers thèmes très représentatifs de la filière équine et de son histoire dans le département. Citons seulement deux ouvrages à titre d'exemple :

- pour l'histoire du trotteur : Gast, Édmond, Le cheval normand et ses origines [...], Paris, Rothschild, c. $1890^{19}$ (fig. n $\left.^{\circ} 11\right)$. 


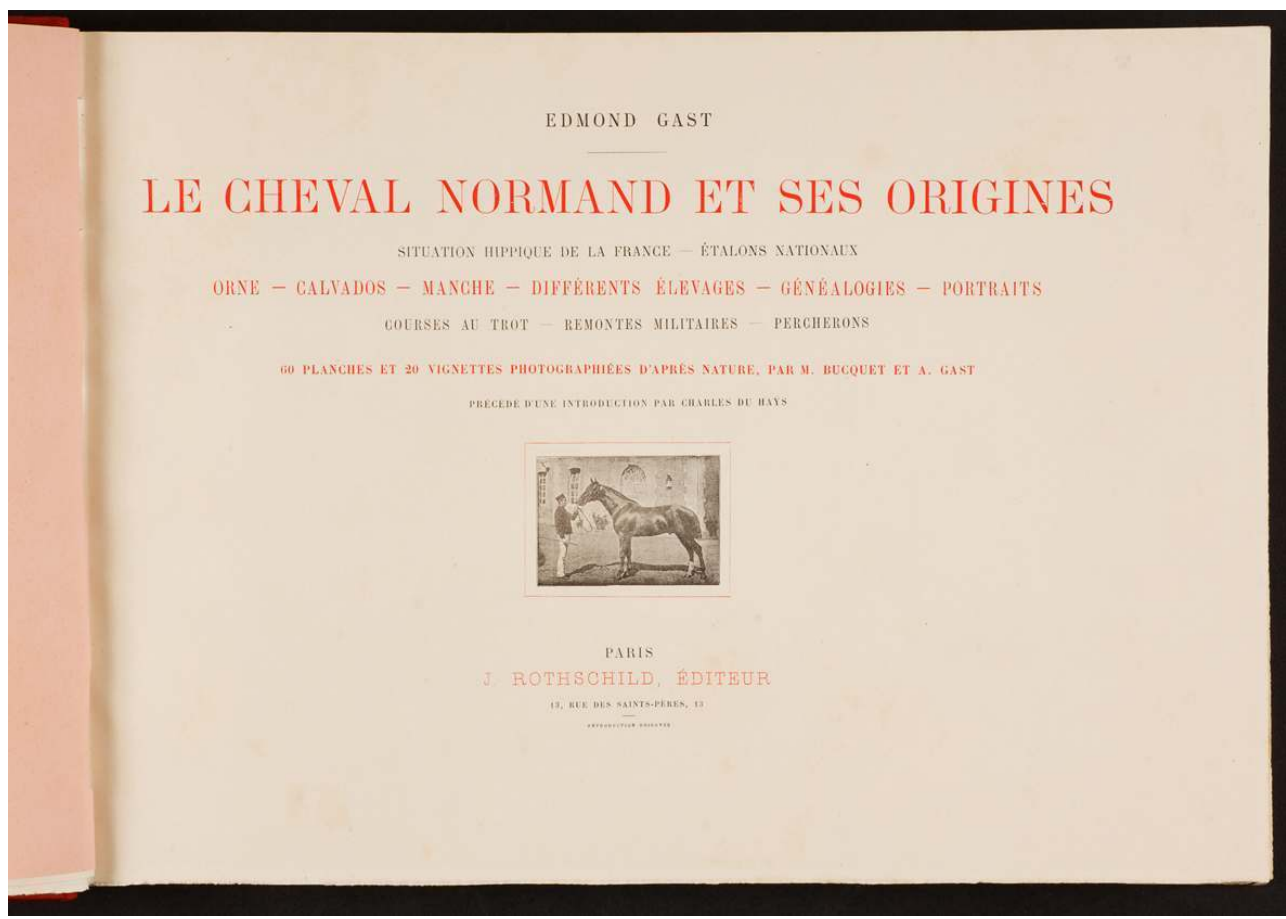

GAST, Edmond. Le cheval normand et ses origines [...]. Paris : Rothschild, c. 1890. AD Manche, BIB RES C 151 .

- pour l'histoire du cheval de selle : [Bizard, Xavier (lieutenant-colonel), préface, Bouvet, Georges, textes rassemblés], Les grands seigneurs, éd. Bouvet, $1951^{20}$ (fig. $\mathbf{n}^{\circ} \mathbf{1 2}$ ).

Figure 12

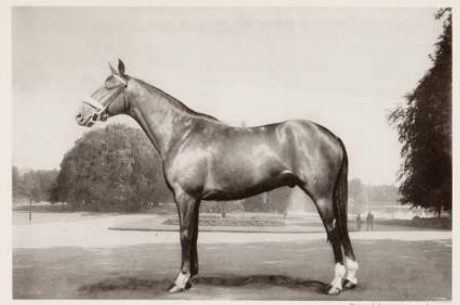

ARLEQUIN D

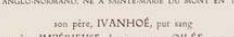

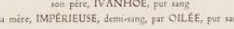

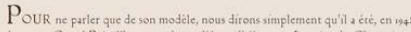

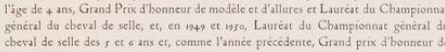

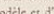

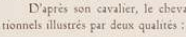

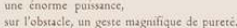

(BIZARD, Xavier (lieutenant-colonel), préface : BOUVET, Georges, textes rassemblés]. Les grands seigneurs, éd. Bouvet, 1951. AD Manche, BIB RES C 221.

Actuellement, ce sont près de 260 monographies (livres et plaquettes catalogués) qui sont consultables aux archives.

La collection des périodiques (15 PER) permet également de compléter les sources archivistiques, voire de les suppléer. Elle est composée :

- de magazines spécialisés (bien évidemment, l'Éperon où presque chaque mois un article est consacré à des élevages, des cavaliers et des chevaux manchois),

- de revues professionnelles (Province courses, par exemple), 
- des annuaires professionnels (Livre généalogique des races françaises de chevaux de selle); des publications officielles (les stud-books),

- des bulletins de différentes associations de chevaux de sport, de trait, de concours d'élevage, des publications de manifestations telles que le Normandie Horse Show, les salons des étalons, les journées Selle Français, etc.

On compte à ce jour près de 190 titres des années 1880 à nos jours dont les collections ne sont pas toujours complètes.

\section{Les archives sonores et audiovisuelles}

Le service des archives sonores a été créé en 2000 afin de compléter le corpus des archives publiques et aussi pour suppléer les destructions de 1944. Les archives de ce service sont constituées de fonds préexistants déposés, donnés et de travaux de collectage réalisés par le service sur des thèmes, des événements et des spécificités manchoises. Le cheval représente une réalité socio-économique majeure qu'il semblait évident de traiter.

Le travail de collecte de témoignages sur le thème du cheval dans le département a débuté en 2002. Il porte plus particulièrement sur le trotteur (monté et attelé). Plusieurs thèmes sont abordés, notamment :

- le cheval comme outil de travail (les mutations liées à la mécanisation, à la motorisation);

- la filière des courses à travers l'élevage, la sélection, la transmission des souches maternelles, celle des savoir-faire à l'entraînement, l'organisation des courses, la formation professionnelle (École de lads-jockeys de Graignes).

En 2005 et 2006, ce même travail a été consacré à la filière du cheval de sport (concours d'obstacles et concours complet). À travers cette filière, de nouvelles activités ont été appréhendées comme l'insémination artificielle, le transfert d'embryon, l'activité des étalonniers privés, la maréchalerie. Plus globalement, c'est également le collectage de cette culture rurale autour du cheval, non académique mais très riche et discrètement codifiée, qui a pu être entrepris. Cette culture empirique porte sur la connaissance encyclopédique des origines des chevaux, notamment des souches maternelles; elle porte sur cette capacité à observer, à évaluer le cheval. Elle touche aussi cette sociabilité issue des rencontres autour des différents événements comme les concours, les foires, les tirages au sort des cartes de saillie, la mise en station de monte de tel ou tel étalon des Haras.

Plus généralement et au-delà des professions, des parcours de vie des personnes interrogées, ce sont des thèmes connexes mais essentiels qui sont évoqués. Le cheval devient alors un formidable support de collectage d'informations auprès de personnes par ailleurs «silencieuses", notamment sur la ruralité, l'agriculture, les réquisitions pendant la seconde guerre mondiale, le Débarquement, la motorisation des machines agricoles, la période de la Reconstruction. Le corpus d'archives sonores sur cette thématique représente environ 60 heures ( 40 heures traitées et 20 encore à analyser).

51 Depuis 2007, le collectage des archives audiovisuelles a tout naturellement été entrepris sans réel traitement documentaire et technique à ce jour. Deux fonds, l'un privé, déposé en 2007 (le fonds Pagnon Productions) et qui couvre trente ans de production (1 300 documents) ainsi que le versement des films de l'ancien studio départemental 
audiovisuel (1 400 documents) permettent d'illustrer sur tous supports (Beta cam, DV cam, VHS, DVD, etc.) :

- l'activité de certains haras privés, le haras de Saint-Lô,

- les concours hippiques, le Grand complet de Martinvast,

- les concours d'élevage des chevaux de sport et de trait, les ventes de chevaux de sport,

- les foires de Gavray et de Lessay,

- les courses hippiques de Graignes,

- certains cavaliers et certains chevaux comme les trotteurs Jag de Bellouet et Général du Pommeau.

Ces documents audiovisuels représentent approximativement une trentaine d'heures d'enregistrements.

\section{Construction des patrimoines et valorisation et réciproquement}

La variété des corpus présentés permet d'appréhender l'ensemble des patrimoines directement ou indirectement :

- le patrimoine immobilier du dépôt de Saint-Lô, des stations de monte par le biais de la documentation textuelle, imprimée et iconographique ;

- le patrimoine mobilier : matériel de travail, de la voiture hippomobile aux instruments agricoles en passant par les instruments de contrôle de l'animal, les instruments et appareillages de portage, de traction par ces mêmes corpus documentaires, les objets relevant de la production artistique, les sculptures, les trophées, les tableaux des étalons et des juments par le biais de couvertures photographiques ou bien de prêts ;

- le patrimoine immatériel grâce aux témoignages oraux prenant en compte les récits de vie, les savoirs, les savoir-faire.

\section{Les réalisations passées}

54 Une exposition a été réalisée en 2006 dans le cadre du bicentenaire des Haras nationaux avec différents partenaires publics et privés. La commémoration de cette création s'imposait alors après la collecte et le classement du fonds.

Ce fonds exceptionnel représente la mémoire de l'activité du haras. Il sert également de trame pour appréhender le travail et le rôle des familles d'éleveurs, les chevaux élevés, sélectionnés, les manifestations sportives et d'élevage organisées afin de valoriser la production, autrement dit la ruralité.

Cette exposition fut également un prétexte, un moyen, afin de collecter auprès des particuliers; des professionnels en activité ou en retraite; auprès des différentes composantes de la filière qui pour une très grande majorité ignoraient l'existence des archives départementales. À l'occasion du vernissage de l'exposition, près de deux cents personnes du monde du cheval ont franchi (très souvent pour la première fois) la porte des archives. Le service des archives a ainsi très concrètement gagné en lisibilité (fig. $\mathbf{n}^{\circ}$ 13). 


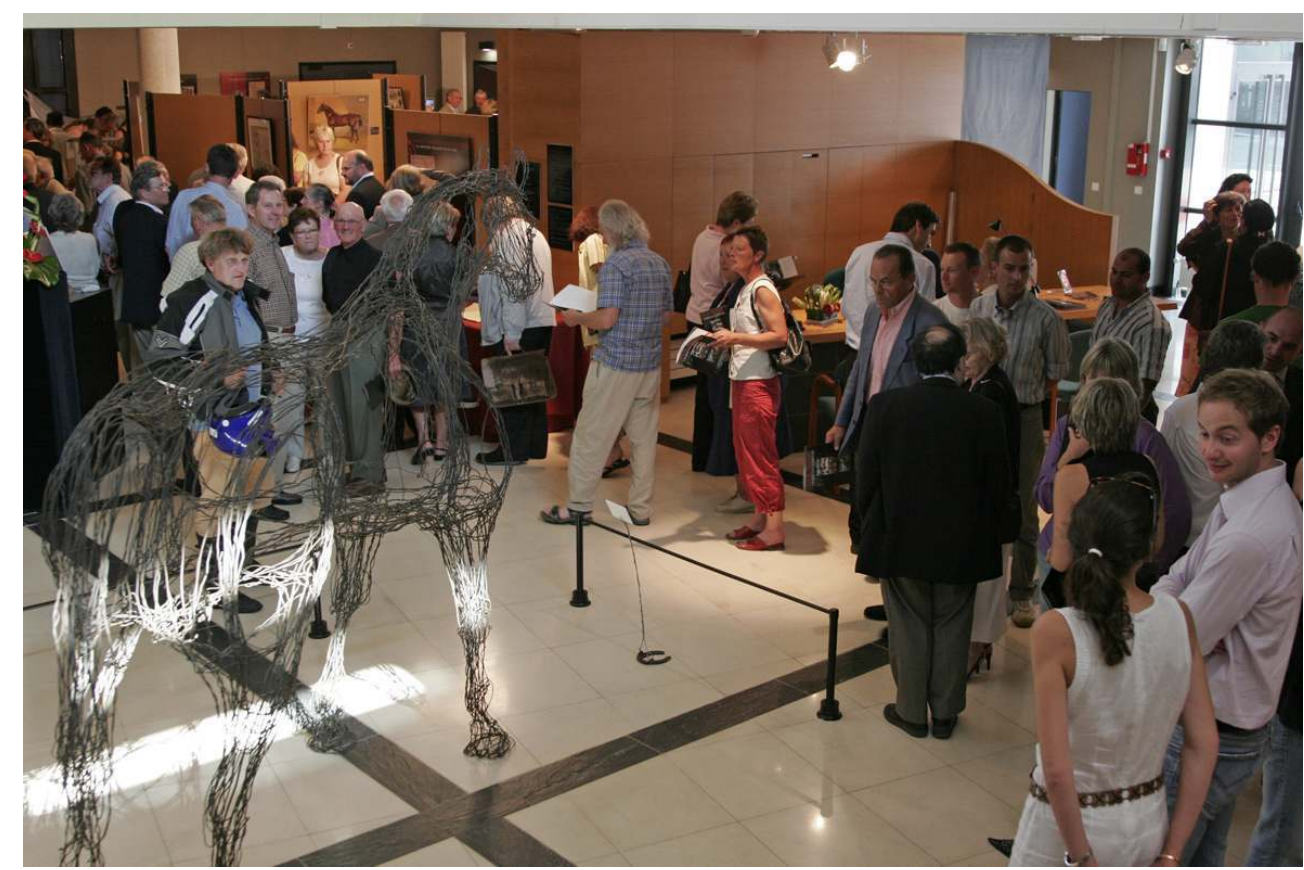

Vernissage de l'exposition « La Manche, terre du cheval » organisée aux archives de la Manche du 7 er juillet au 10 novembre 2006.

Phot. A. Poirier, 3 Num 2006/478. ( ) AD Manche/CG50.

Ce projet a aussi conditionné la réussite du collectage des archives sonores en donnant un sens, un but aux entretiens. Simultanément, le travail du service des archives sonores a permis sur le terrain la découverte, la connaissance et le collectage indirect (quelque peu déguisé) d'un patrimoine mobilier en mains privées qui fait défaut au sein des collections publiques. Par exemple, les personnes interviewées ont quasi systématiquement prêté des photographies (tout au moins dans le monde du cheval de sport, moins secret que le microcosme des trotteurs), photographies très intéressantes pour documenter l'histoire de la ruralité, des sociétés hippiques rurales et des concours hippiques dans les années cinquante et soixante (fig. $\left.\mathbf{n}^{\circ} \mathbf{1 4}\right)$. Certains objets ont également pu être photographiés (des trophées, des tableaux, des matériels, etc.). 
Figure $^{\circ} 14$

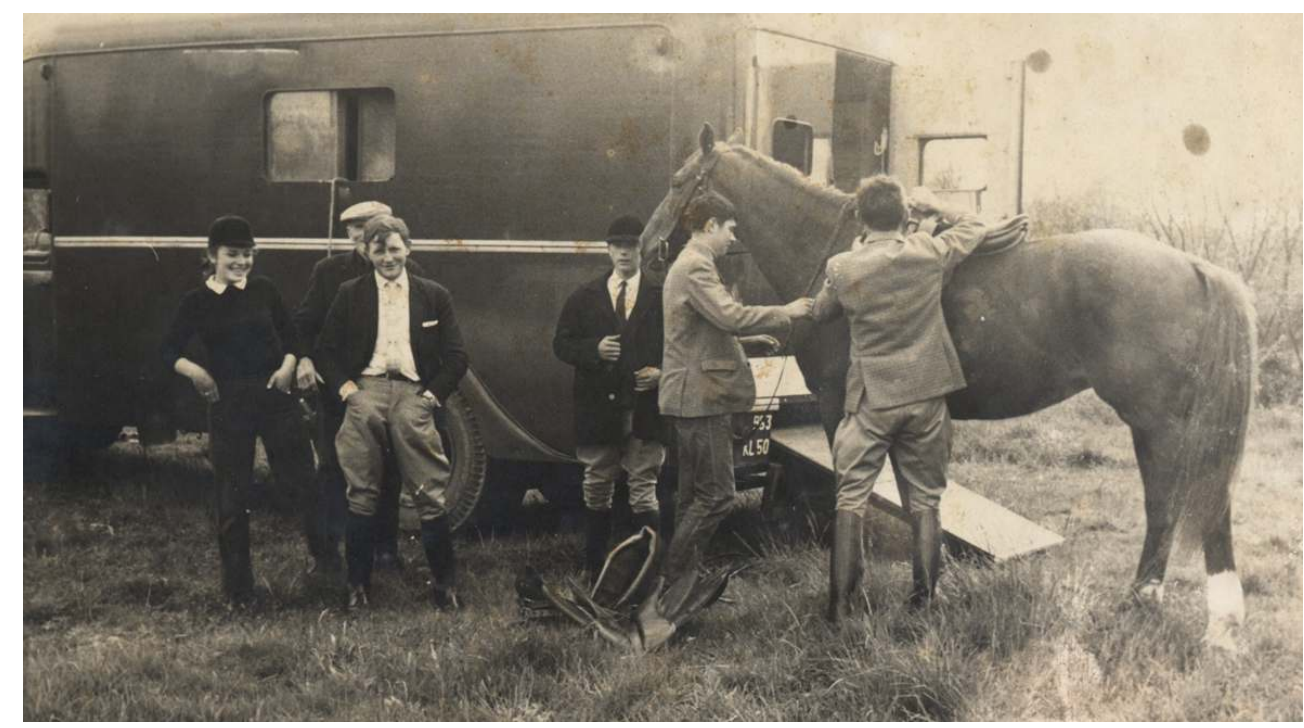

Cavaliers de la société hippique rurale de Cerisy-la-Salle au concours de Périers, $7^{\text {er }}$ mai 1968.

Phot. A. Poirier, 84 Num 242. (c) AD Manche/CG50.

La communication et l'action culturelle des archives départementales ont joué jusque-là dans ce domaine un rôle primordial: il faut être connu, voire être reconnu afin d'augmenter les programmes de collecte des archives privées. Cette communication depuis les années 2000 a été mise en forme par le biais des expositions, des publications propres, voire des partenariats mis en place avec différents acteurs dans le domaine patrimonial et culturel. Elle est orientée de plus en plus vers le numérique, le multimédia, l'internet.

Il ne nous appartient pas de dire que les expositions et les manifestations qui accompagnent les actions des archives sont intéressantes mais elles sont en tout cas intéressées. C'est un aspect qui peut paraître, vu de l'extérieur, de la part des publics ou bien de la profession, comme anecdotique, mais il procède très concrètement de la politique de reconstitution des collections. C'est un enjeu patrimonial essentiel. La valorisation par le biais de la communication des documents sous toutes ses formes est à la fois une fin, mais aussi un moyen d'enrichir le patrimoine, les patrimoines, dont les patrimoines équestres et équins.

\section{Les axes de travail pour le futur}

\section{Sur le plan archivistique, documentaire et infodocumentaire}

Poursuivre la collecte d'archives associatives s'impose : les fonds des sociétés de courses, des sociétés hippiques rurales (celle de Sainte-Mère-Église a été fondée en 1933), des associations organisatrices de concours complets ${ }^{21}$, des concours d'élevage, organisatrices de concours d'attelage, mériteraient d'être sauvegardés.

61 La duplication des archives du haras de Torigni-[sur-Vire] (sous forme de microfilmage ou de numérisation) conçu par Honoré III Grimaldi dans les années $1760^{22}$ et conservées à Monaco ${ }^{23}$ serait également un nouvel apport important pour la période de l'Ancien Régime beaucoup moins bien documentée. 
62 À l'heure du web 2.0, du web des données, du web sémantique et collaboratif, la création de portails numériques thématiques sur le cheval et la guerre, sur le cheval et la mer, sur le cheval et l'agriculture, etc., peut et doit représenter désormais pour les services culturels et patrimoniaux, une priorité. La production, à court et moyen terme, des données descriptives de manière interopérable (en .xml) devrait permettre de partager et de cumuler des données sur des patrimoines multiformes et complémentaires.

De plus, la possibilité d'utiliser des modèles conceptuels de description, pour l'ensemble des patrimoines, semble primordiale. Le projet d'harmonisation de la production des données culturelles et patrimoniales au sein du ministère de la Culture représente à ce titre un enjeu essentiel. À partir des normes et des structures de contenu mises en œuvre par les archives, les bibliothèques, les musées, l'archéologie, l'inventaire, il s'agit de tenter de définir une carte d'identité commune aux biens culturels.

\section{Dans le domaine des témoignages oraux}

Poursuivre plus ponctuellement le collectage de témoignages sur une filière qui voit apparaître de nouveaux acteurs dans un contexte de mutation sociale, économique et professionnelle actuellement très importante, à l'aune des changements survenus aprèsguerre, notamment dans les pratiques du cheval de loisir par des publics de moins en moins ruraux. Des échanges, des collaborations avec les établissements d'enseignement professionnel (Graignes, Montebourg, Saint-Hilaire-du-Harcouët) permettraient d'enrichir le corpus des témoignages et pourraient fournir des supports pédagogiques aux élèves.

Ces opérations de collectage offriraient, à nouveau, l'opportunité d'enrichir la couverture photographique du patrimoine mobilier privé (voire immobilier) qui peut en découler.

\section{Dans le domaine de l'Inventaire}

Pour revenir au territoire manchois, un projet de couverture photographique des stations de montes, disséminées sur le territoire (par le photographe des archives) semble maintenant important à mettre en place. Il ne s'agit pas de concurrencer les collègues du service régional de l'Inventaire de Caen mais de photographier un patrimoine (presque) vernaculaire dont la conservation matérielle, lorsque ces bâtiments existent encore, peut paraître fragile. Pour le Haras de Saint-Lô, cette campagne a été réalisée lors de la préparation de l'exposition de 2006.

67 On pourrait également envisager, dans le domaine des courses, une campagne photographique des hippodromes et de leurs installations très peu représentés à ce jour dans les fonds photographiques.

\section{Valorisation d'un patrimoine archivistique, patrimoine génétique}

Enfin, et en guise de conclusion, un aspect très important qui n'a pas encore été explicité dans le champ patrimonial doit être souligné. Il s'agit, au-delà du patrimoine archivistique, du patrimoine génétique, et du patrimoine économique qui en découle.

À la saison creuse, avant le stress du poulinage et les espérances parfois folles de la monte, certains éleveurs (étrangers en général) viennent consulter à Saint-Lô la collection des carnets de saillies. Ces généalogistes d'un genre particulier ont fait 
l'acquisition de juments Selle Français. Ils veulent connaître "le papier » de leurs poulinières pour des raisons commerciales et économiques. Ils souhaitent inscrire dans le temps la filiation de leurs propres produits et en tirer profit.

Dans ce cas précis, l'utilisation du patrimoine archivistique équin est très utilitariste et pas du tout patrimoniale, tournée vers le passé, vers un âge d'or des chevaux anglonormands, ou bien de leurs merveilleux descendants Selle Français. C'est là une autre forme de patrimoine - génétique - qui lui aussi est en perpétuelle évolution.

71 Un patrimoine génétique, qu'en son temps, Pierre-Étienne Joseph-Lafosse a perçu, pensé, utilisé avec génie, avant les autres.

\section{NOTES}

1. - Lettre conservée en main privée. Le clos Grenneville est situé devant la propriété de l'auteur.

2. - Pierre-Étienne Joseph-Laffosse (1828-1897), de Saint-Côme-du-Mont (canton de Carentan). Elisa, après une seule année de courses, en 1856, où elle arrive toujours première, est mise à la reproduction et, d'une fertilité rare, elle engendre dix-neuf produits dont le célèbre Conquérant en 1858. Pierre-Étienne Joseph-Lafosse se sépare de son haras en 1869 ; seule Élisa n'est pas vendue, ce qui souligne l'attachement qu'il porte à cette jument. Grâce à l'excellence de ses produits, plusieurs de ses parents vont pousser haut les couleurs du trotting français. Ce sont Jules Lecuyer, son neveu de Saint-André-de-Bohon, naisseur de Phaëton, Émile Allix-Courboy et Jean-Adolphe Gosselin, ses cousins germains de Saint-Côme-du-Mont, naisseurs de Tigris et de Fuchsia. À la vente de son cheptel figurent d'autres grands noms de l'élevage : Gislain, CeranMaillard, Buhot, Touzard et Mériel. Joseph-Lafosse a été, par la suite, un éleveur reconnu et un prix portant son nom est couru chaque automne à Vincennes. Le texte qu'il consacre à la mort d' Élisa est très représentatif de la relation qui peut exister entre l'éleveur et ses chevaux.

3. - ROCHE, Daniel. La culture équestre occidentale, XVI ${ }^{e}-X_{X}{ }^{e}$ siècle, l'ombre du cheval, tome 1, le cheval moteur. Paris : Fayard, 2008, 479 p.

4. - DIGARD, Jean-Pierre. Une histoire du cheval : art, technique, société. Arles : Actes Sud, 2004, 230 p.

5. - Archives départementales de la Manche (AD Manche), $213 \mathrm{~J} 70$, fonds Paul de Gibon : « C'est le département de la Manche seul qui fournit aujourd'hui en chevaux de remonte 80 p. 100 de l'effectif total, c'est-à-dire que 80 p. 100 de ces chevaux de remonte sont nés dans le département de la Manche, ainsi que l'attestent les extraits de naissance réclamés aux vendeurs ». Rapport du sénateur Damecour sur le cheval de demi-sang et la remonte, $2^{\mathrm{e}}$ séance du 14 décembre 1926.

6. - L'établissement appelé par l'ensemble de ses usagers " haras de Saint-Lô », est en réalité un dépôt d'étalons. Ceux-ci sont affectés pendant la saison de monte dans les stations du même nom, réparties sur le territoire de la circonscription de Saint-Lô. L'appellation de haras doit être réservée aux établissements rassemblant des étalons et des juments. Par souci de simplification, nous le citerons sous l'appellation courante de « haras de Saint-Lô ».

7. - Mention des races ou des produits identifiés selon leur usage: race normande, anglonormande, de demi-sang, carrossière, d'attelage, anglais, anglais de carrosse, de demi-sang, de pur sang, de selle, de chasse; des genres : selle, postier, cob, cob-normand, distinction du selle et du cob par la longueur de la queue, etc. 
8. - AD Manche, 1 Mi 548 (R 1) : projet d'établissement du haras : correspondance, rapports, an VIII - 1815, F10/715 ; mouvements des étalons, 1811-1820 ; montes, 1814-1820 ; établissement dans l'abbaye [de Sainte-Croix-de-Saint-Lô], réparations, constructions, 1806-1820, F 10/1126 ; $1 \mathrm{Mi} 548$ (R 2) : inspections, 1806-1820, F 10/786, inspections, 1821-1831, F 10/1023, inspections, 1832-1838, F 10/1034.

9. - Les bombardements de Saint-Lô au début du mois de juin 1944 ont détruit partiellement « le vieux haras" aménagé au XIX siècle autour de l'abbaye Sainte-Croix. Au "nouveau haras", comme on l'appelait à l'époque, les bombes détruisent la sellerie, les écuries 1 et 2 , ainsi que la maison dite de l'adjudant. Elles endommagent l'écurie $n^{\circ} 6$, le pavillon du concierge, la porte Est qui ne sera pas reconstruite, ainsi que le manège. Pour l'institution saint-loise, ce sont 70 étalons qui ont été tués ou réquisitionnés. À cette époque, la plupart des étalons ne sont pas dans les écuries bombardées mais en activité dans les différentes stations de monte dans le ressort de la circonscription ce qui a réduit considérablement les pertes.

10. - Un chartrier est un fonds d'archives produites et reçues dans le cadre de la gestion des biens d'une famille ou d'une institution depuis la fin du Moyen Âge et surtout sous l'Ancien Régime. Par extension, y sont amalgamées les archives dites familiales, domaniales et seigneuriales.

11. - Série des documents entrés par voie extraordinaire depuis 1944 selon le cadre de classement des archives départementales.

12. - 97 chevaux sont identifiés par la robe : 23 chevaux «noirs"; 23 chevaux bais de "brun bay » à « cler bay »; 21 chevaux « liarts » (gris et gris pommelés) ; 15 " moreaux » (noirs foncés et luisants) $; 12$ «fauves » (tirant sur le roux) 3 chevaux « saures» (jaune tirant sur le brun). Pour une meilleure distinction, certains sont dits « estellés».

13. - AD Manche, chartrier de la baronnie de Bricquebec, $280 \mathrm{~J}, 1283-1842$.

14. - AD Manche, chartrier du Mesnil-Vitey, 114 J C 5 : procédure relative au jardin de la Pierre et au grand herbage à Airel, 1753-1758.

15. - AD Manche, tribunal de première instance de Valognes, 3 U 6/653, procès-verbaux des gardes forestiers de la commune de Bricquebec (1791-1794).

16. - Gilles Picot, sieur de Gouberville, dont le très célèbre journal couvrant la période 1549-1562 est aussi riche d'informations sur le cheval, ses usages dans le Cotentin rural du XVI ${ }^{\mathrm{e}}$ siècle. Voir notamment ROCHE, Daniel. «Les chevaux de Gouberville : Élevage et usages dans la Normandie du XVI ${ }^{\mathrm{e}}$ siècle ». Les cahiers goubervilliens, 2010, $\mathrm{n}^{\circ} 14,24 \mathrm{p}$.

17. - GARCIN, Jérôme. La chute de cheval. Paris : Gallimard, 1998, 142 p.

18. - GOURAUD, Jean-Louis. Hippomanie. Lausanne : Favre, 2011, 687 p.

19. - AD Manche, BIB RES C 151.

20. - AD Manche, BIB RES C 221.

21. - Celle de Martinvast a organisé des épreuves Coupe du monde avant de gagner le site du Haras du Pin depuis 2010. Elle doit son succès à la compétence et au travail de toute la famille Le Goupil et notamment à l'expérience de M. André Legoupil. Il fut le premier civil champion de France en complet en 1961 et deux fois cavalier olympique dans cette discipline (Tokyo en 1964 et Mexico en 1968).

22. - Dès la fin de la guerre de Sept ans, Honoré III achète des chevaux anglais. En 1768, un an avant Voyer d'Argenson, il découvre l'Angleterre et adopte avec admiration et passion les principes d'élevage et de courses à l'anglaise. À son retour en France, il dépense des sommes considérables pour le domaine de Torigni.

23. - En 1715, Jacques-François-Léonor de Matignon (Jacques IV de Matignon) épouse LouiseHippolyte Grimaldi, héritière de la principauté de Monaco, et devient prince de Monaco sous le nom de Jacques $\mathrm{I}^{\mathrm{er}}$. 


\section{RÉSUMÉS}

Pour des raisons historiques, géographiques, économiques, le département de la Manche est l'un des plus concernés par l'histoire et le patrimoine lié au cheval. Les archives départementales ont été totalement sinistrées en 1944. Archives du haras de Saint-Lô, archives privées, archives sonores, imprimés, iconographie sont autant de supports d'un patrimoine équin et équestre multiforme. Ce même patrimoine devient aussi un enjeu majeur pour la reconstitution des collections. La collecte, la gestion archivistique et documentaire de l'ensemble des matériaux constitutifs de ce patrimoine matériel et immatériel, sa construction sont essentielles pour la valorisation, la connaissance de ce territoire.

For historic, geographical, economic reasons, the department of La Manche is one of the most concerned by the history and the heritage about horse. Departmental records office was totally destroyed in 1944. Archives of the "National stud horse » of Saint-Lô, private archives, sound archives, printed matters, iconography are so many supports of a equine and equestrian multiform heritage. Heritage becomes also a major issue for the restore of collections. The collecting, the archival and documentary management of all the constituent materials of these material and immaterial heritage, his construction are essential logic for the valorization, the knowledge of this area.

\section{INDEX}

Keywords : Archival and documentary heritage equine of "La Manche": constitution and restore; construction and valorization of the heritages, and mutually

Mots-clés : archives publiques, archives privées, archives sonores, archives audiovisuelles, iconographie, imprimés, bibliothèque, documentation, cheval de sport, cheval de course, cheval de guerre, destruction, Selle Français, Trotteur, Saint-Lô, Pierre-Étienne Joseph-Laffosse, Haras de Saint-Lô

\section{AUTEUR}

\section{ALAIN TALON}

directeur adjoint des archives départementales et du patrimoine culturel de la Manche alain.talon@manche.fr 